\title{
Interactions between a luminescent conjugated oligoelectrolyte and insulin during early phases of amyloid formation
}

Jens A. Wigenius, Gustav Persson, Jerker Widengren and Olle Inganäs

\section{Linköping University Post Print}

N.B.: When citing this work, cite the original article.

This is the author's version of:

Jens A. Wigenius, Gustav Persson, Jerker Widengren and Olle Inganäs, Interactions between a luminescent conjugated oligoelectrolyte and insulin during early phases of amyloid formation, 2011, Macromolecular Bioscience, (11), 8, 1120-1127.

http://dx.doi.org/10.1002/mabi.201100016

Copyright: Wiley-VCH Verlag Berlin http://www.wiley-vch.de/publish/en/

Postprint available at: Linköping University Electronic Press

http://urn.kb.se/resolve?urn=urn:nbn:se:liu:diva-54898 
DOI: 10.1002/mabi.201100016

Article type: Fullpaper

\section{Interactions between a luminescent conjugated oligoelectrolyte and insulin during early phases of amyloid formation}

Jens Wigenius, Gustav Persson, Jerker Widengren and Olle Inganäs*

Dr. J.Wigenius and Prof. O.Inganäs corresponding authors*

Biomolecular and Organic Electronics, Department of Applied Physics, IFM, Linköping University, SE-581 83 Linköping, Sweden,

E-mail: ois@ifm.liu.se

Dr. J.Wigenius Current address: Department of Chemical and Biological Engineering, Chalmers technical University, SE-412 96 Gothenburg, Sweden

E-mail: jens.wigenius@chalmers.se

Dr. G.Persson and Prof. J Widengren

Experimental Biomolecular Physics, Department of Applied Physics, Royal Institute of Technology, SE-106 91 Stockholm, Sweden

E-mail: gup@kth.se and, jwideng@kth.se

Aggregates of misfolded proteins play an important role in diseases such as Alzheimer's. We here demonstrate how the anionic oligothiophene p-FTAA interacts with and influences prefibrillar protein assemblies during the earlier stage of in vitro fibrillation. Conjugated polythiophenes have previously been demonstrated to detect and discriminate between different types of protein aggregates and also introduce luminescent or conductive properties to these nanoscale fiber structures. We use fluorescence spectroscopy, dynamic light scattering, transmission electron microscopy and fluorescence correlation spectroscopy to follow the interplay between p-FTAA and insulin during in vitro fibrillation.

\section{Introduction}


The field of organic electronics is expanding rapidly, and the pursuit of miniaturizing and organizing conjugated polymers (CPs) has found new routes (see $\operatorname{Ref}^{[1]}$ for a recent review). Biomolecular nanotemplates can be given electronic and photonic properties through selfassembly in the presence of these polymers. One of the most interesting classes of biological macromolecules for self-assembly of advanced composite nanostructures is proteins. They offer an enormous variation of complexity, functionality and structural properties. The folding of an amino acid polypeptide chain into its three-dimensional native protein structure is crucial for its biological activity. However, this folding process can also be sensitive to various perturbations. Partially unfolded and/or misfolded proteins may randomly transform into small spherical pre-fibrillar aggregates that organize into short thin structures known as protofilaments, and further assemble into highly ordered mature fibrils, so called amyloids. ${ }^{[2,}$ ${ }^{3]}$ Thus a stable nanowire with dimensions up to $10 \mu \mathrm{m}$ in length and about 7 to $10 \mathrm{~nm}$ in diameter can be obtained. ${ }^{[4-6]}$ A number of thiophene based CPs show high affinity for amyloids $^{[7-9]}$ and have been assembled into conducting or luminescent molecular wires, ${ }^{[10-12]}$ or integrated into OLEDs and organic solar cells to enhance efficiency. ${ }^{[13,14]}$ The interaction between thiophene based CPs and amyloids is not fully understood, but correlates with ionic interactions, hydrogen bonding and hydrophobic interactions ( $\pi-\pi$ stacking). Furthermore, interactions between the repetitive $\beta$-sheet structure of amyloids and the hydrophobic backbone of the polyelectrolyte are possibly very important. ${ }^{[15,16]}$ In this regard, it is of great importance to understand the molecular interactions between the pre-fibrillar states and the $\mathrm{CP}$, as this understanding is essential for the design of novel amyloid ligands. To better understand the kinetics of the pre-fibrillar amyloid formation and the molecular interactions between $\mathrm{CP}$ and bovine insulin, we utilized dynamic light scattering (DLS), fluorescence correlation spectroscopy (FCS), transmission electron microscopy (TEM) and steady-state photoluminescence of a conjugated oligoelectrolyte (COE). Fibrillation of bovine insulin under acidic conditions was used as a model system, as it was recently reported that the 
formation of insulin amyloid fibrils is preceded by the formation of a hexameric helical nucleus of insulin under these conditions. ${ }^{[17]}$ We used a novel COE, the pentameric oligothiophene derivative p-FTAA (Figure 1), recently developed by Nilsson and co-workers for in vivo optical imaging of amyloid deposits, ${ }^{[9]}$ and for identification of pre-fibrillar nonthioflavinophilic $\left(\mathrm{Th}-\mathrm{T}^{-}\right)$protein aggregates that occur at an early stage of protein aggregation. ${ }^{[18]}$ The identification and characterization of such pre-fibrillar states is of particular interest both because of their likely role in the mechanism of fibril formation and the increasing awareness that these species are likely to play a critical role in the pathogenesis of protein misfolding diseases. ${ }^{[19-21]}$ Finding a way to earlier stage diagnosis of these diseases is of great importance.

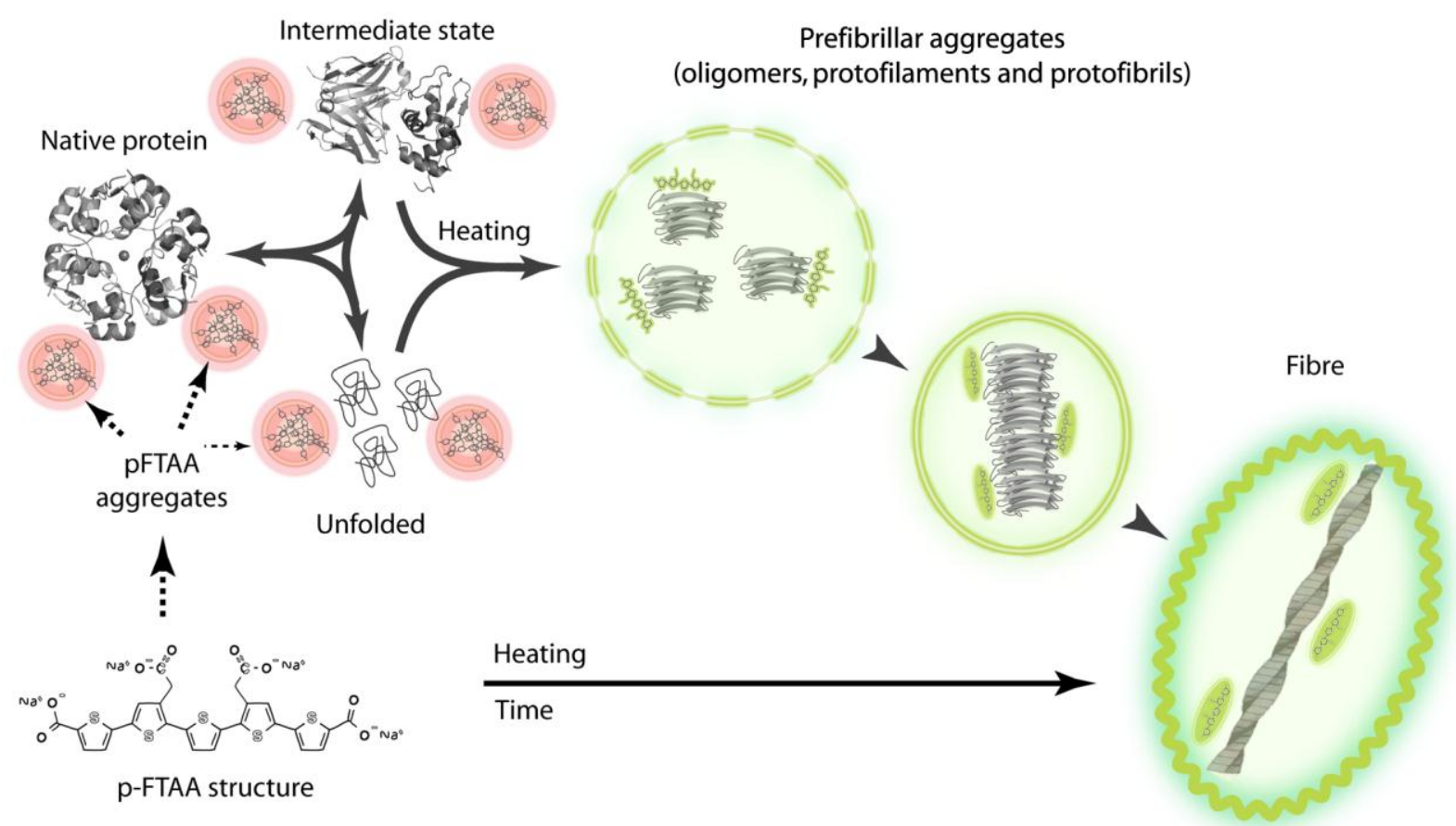

Figure 1. Sketch of suggested insulin folding and aggregation pathways under acidic conditions, as described in the literature and used in this study. Also included are the expected COE interactions, with the different states of the protein and the predicted fluorescence response of p-FTAA illustrated by colors in the different states. Bottom left: Chemical structure of p-FTAA. Protein molecular structure obtained via Polyview-3D. ${ }^{[33]}$

Our studies verify that p-FTAA interacts with pre-fibrillar aggregates of insulin, preceding the formation of mature insulin amyloid fibrils. However, under the present conditions, the 
formation kinetics and the structure of these pre-fibrillar species are affected by the presence of p-FTAA.

\section{Experimental Section}

The synthesis of p-FTAA (figure 1) has been reported elsewhere. ${ }^{[9]}$ The compound was diluted to a stock concentration of $1.5 \mathrm{mM}$ in deionized water (mQ water) (Millipore 18.2 $\mathrm{M} \Omega$ ), aliquoted and stored protected from light at $-20^{\circ} \mathrm{C}$. On the day of use the stock solution was further diluted in $\mathrm{mQ}$ water to $15 \mu \mathrm{M}$ before addition to the sample reaching a final sample concentration of $0.2 \mu \mathrm{M}$, if not stated otherwise in the text.

All fibrillation experiments were performed following a protocol reported elsewhere. ${ }^{[17]}$ In brief, a solution containing bovine insulin (Sigma-Aldrich, Sweden) in $2 \mathrm{M}$ acetic acid (HAc) and $0.5 \mathrm{M}$ sodium chloride $(\mathrm{NaCl})$ was prepared. After filtration $(0.2-\mu \mathrm{m}$ HAWP (Millipore) syringe filter) the insulin concentration was determined and adjusted to $5 \mathrm{mg} \cdot \mathrm{ml}^{-1}$, measuring the absorbance at $280 \mathrm{~nm}$ using $\varepsilon 280=5480 \mathrm{M}^{-1} \cdot \mathrm{cm}^{-1}$ as extinction coefficient. The solution was divided into two samples, one with addition of p-FTAA $(0.2 \mu \mathrm{M})$ and the second without, adjusting the volume with $\mathrm{mQ}$ water. Fibrillation was enhanced by heating the solution to $50^{\circ}$ C (Figure 1).

\section{Luminescence characterization:}

Aliquots from the fibrillation process were removed, at regular time intervals, and rapidly cooled down. A small volume of p-FTAA was added to the pure insulin sample to reach 0.2 $\mu \mathrm{M}$ p-FTAA concentration, negligibly affecting the insulin concentration. Emission and excitation spectra were measured with a plate reader, (Safire2 (TECAN, Switzerland), using Costar 96 half-width, flat bottom, black non-treated plates (Corning, UK)). 450-nm light was used for excitation, averaging three individual samples and subtracting the buffer response.

\section{Dynamic light scattering:}

Fibrillation kinetics of the two types of samples was studied and hydrodynamic radii $(\mathrm{Rh})$ of p-FTAA/insulin complexes, or insulin only, were determined by dynamic light scattering (DLS) as average results of three to five individual measurements. The DLS setup, an ALV/DLS/SLS-5022, compact goniometry system (ALV Gmbh, Langen, Germany), used a HeNe-laser (632 nm, $22 \mathrm{~mW}$ ) as light source and two avalanche photo diodes (PerkinElmer, Vaudreuil, Quebec, Canada) as detectors, working in cross-autocorrelation mode. The scattered light was collected at $90^{\circ}$ from the incident laser. The temperature was kept constant $\left(50.00 \pm 0.05^{\circ} \mathrm{C}\right)$ in the surrounding toluene bath. Intensity correlation curves were analyzed with the ALV-500/E/EPP\&ALV 60X0-win V3.0.2.3 software based on standard CONTIN analysis. ${ }^{[22]}$ All solutions were filtered $(0.2 \mu \mathrm{m})$ and handled in a clean environment to minimize dust contamination.

\section{Transmission electron microscopy:}

The samples were examined in a Philips CM200 TEM at $120 \mathrm{kV}$. The sample solution $(20 \mu \mathrm{l})$ was placed on carbon-coated copper grids for 2 minutes. The sample was blotted dry using a filter paper, to remove excess solution, and rinsed with $\mathrm{mQ}$ water. Thereafter, they were and negatively stained with $2 \%$ uranyl acetate $(\mathrm{W} / \mathrm{V})$ in $\mathrm{mQ}$ water, again blotted dry and rinsed.

\section{Fluorescence correlation spectroscopy:}

Fluorescence correlation spectroscopy $(\mathrm{FCS})^{[23,24]}$ measurements were performed on a homebuilt confocal microscope. ${ }^{[25]}$ The samples were applied as hanging droplets and excited by a 440-nm diode laser (LDCU12/6104, Power Technology, Inc., Little Rock, AR, USA). The excitation power was controlled by insertion of neutral density glass filters into the beam path. A Zeiss $63 \times$, NA 1.2, NeoFluor water immersion objective was used for focusing of the excitation light and for fluorescence collection in an epi-illumination arrangement. The 
fluorescence emission was separated from the excitation light by a dichroic mirror and a 50$\mu \mathrm{m}$ pinhole was placed in the back focal plane to limit the axial extension of the observation volume. After the pinhole the fluorescence was split by a 50/50-beam-splitter cube, discriminated from scattered light by optical band-pass filters (HQ565/75m, Chroma Technology, Rockingham, VT, USA) and focused onto two single-photon sensitive avalanche photodiodes (APDs, SPCM-AQR-13/14, PerkinElmer Optoelectronics, Fremont, CA, USA) in a so called Hanbury-Brown and Twiss arrangement. ${ }^{[26]}$ This arrangement, in combination with cross-correlation, circumvents the detector dead-time and abolishes the effects of detector after-pulsing on the correlation curves, thus enabling the use of the full timeresolution of the correlator (ALV-6010/160, ALV GmbH, Langen, Germany). Calibration measurements using a well known emitter, rhodamine 110, were initially performed to align the setup and determine the properties of the observation volume, and were then repeated several times in between the p-FTAA measurements. The fibrillation samples were prepared in similar manner as for luminescence characterization described above.

\section{Results and Discussion}

As previously demonstrated the p-FTAA emission profile can be used to visualize nonthioflavinophilic ( $\left.\mathrm{Th}^{-} \mathrm{T}^{-}\right)$pre-fibrillar states during the amyloid formation of both recombinant $\mathrm{A} \beta$ peptide and bovine insulin $(\mathrm{BI}) .{ }^{[9,18]}$ In this work we used $\mathrm{BI}$ as model system since the fibrillar process have been thoroughly described, ${ }^{[17]}$ and followed the fibrillation event by dynamic light scattering and TEM. Two types of samples were analyzed, the first with BI only and the second with p-FTAA added, and heated to $50^{\circ} \mathrm{C}$ in order to accelerate the fibrillation process (see experimental section for details). In the sample with BI only, small diffusing scattering units were initially observed (Figure 2a) corresponding to a hydrodynamic radius $(\mathrm{Rh})$ of $1.5 \pm 0.5 \mathrm{~nm}$, as expected from a pure insulin dispersion (Figure 2b). The fibrillation event is clearly identified as a shoulder rising in the later part of the autocorrelation function, visible already after 15 minutes of heating, indicating that pre-fibrillar oligomeric states of insulin have formed at this time. This is also reflected by broad distributions with increasing size of scattering objects in the CONTIN analysis of the correlation function (Figure 2b). The auto-correlation function continuously shifts to longer 

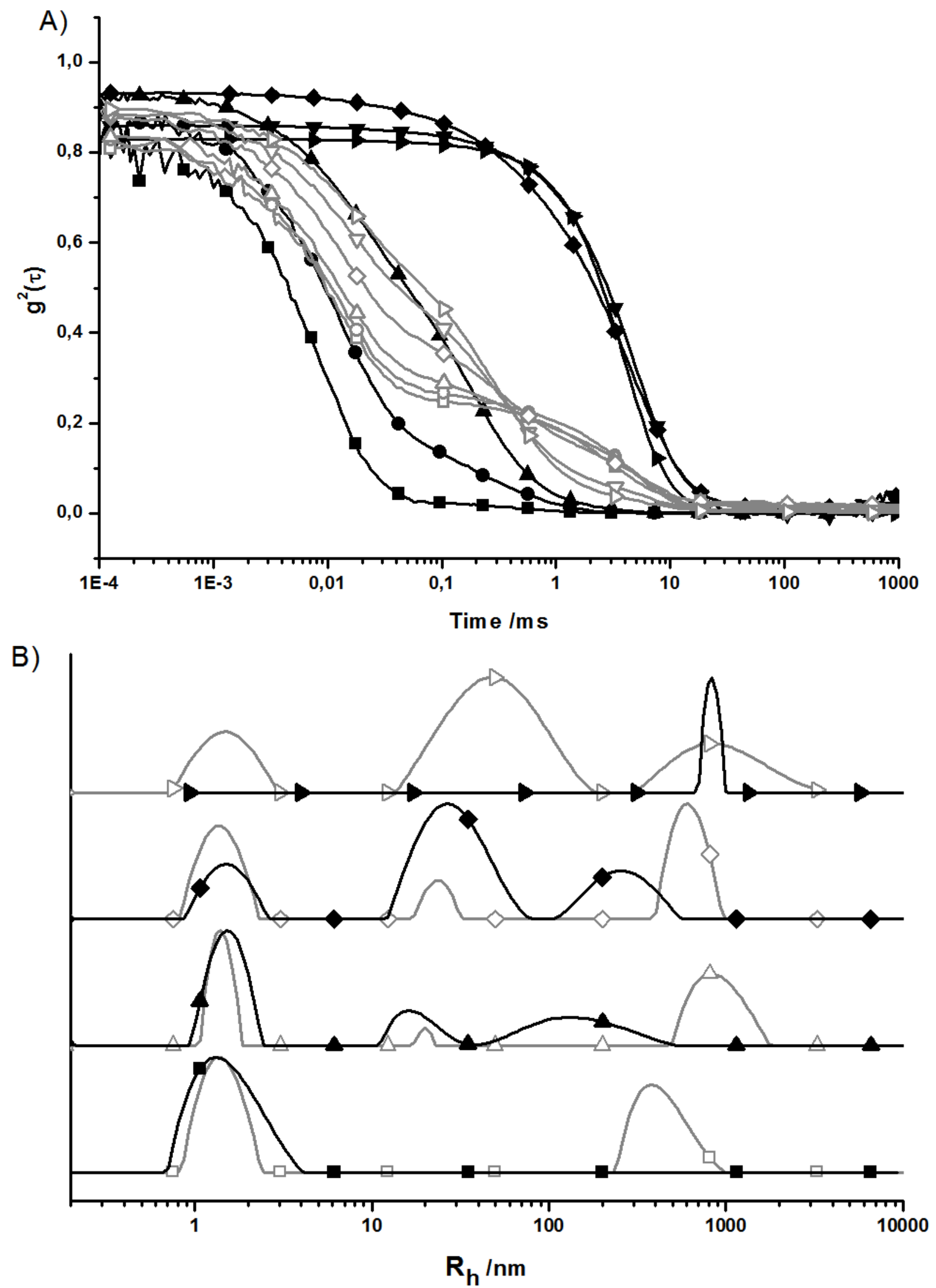

Figure 2. A) DLS auto-correlation functions, open, grey symbols represent measurements with p-FTAA present during the fibrillation process. The corresponding solid, black symbols represent measurements during the fibrillation process in the absence of p-FTAA at 0 minutes $(\boldsymbol{\bullet}, \square),(\bullet, \circ) 15$ minutes, $(\boldsymbol{\Delta}, \triangle) 30$ minutes, $(\bullet, \diamond) 60$ minutes, $(\boldsymbol{\nabla}, \nabla) 90$ minutes and $(\triangleright, \triangleright) 120$ minutes after the sample was placed in the sample holder and heated to $50^{\circ} \mathrm{C}$. 
B) Four of the corresponding peak-normalized size distributions of insulin and insulin aggregates calculated with CONTIN analysis, starting from the bottom curve 0, 30, 60 and 120 minutes after start.

diffusion times as heating continues, with a stretched decay indicating a wide size distribution. After 60 minutes a steep shape is regained and after 60 additional minutes, one sharp peak with Rh between $800 \mathrm{~nm}$ and $1.1 \mu \mathrm{m}$ in the CONTIN analysis is seen. Small precipitates start to sediment after a longer time period, complicating the interpretation of light scattering data, rendering DLS inappropriate for further study of later stages of the fibrillation event.

A different situation is observed with p-FTAA present in the solution during the fibrillation process. Already in the initial measurement a distinct shoulder is observed in the autocorrelation function, corresponding to an Rh between $300 \mathrm{~nm}$ and $400 \mathrm{~nm}$, along with a more rapidly decaying component similar to the one noted from single insulin proteins in the first sample. The larger complexes could originate from p-FTAA aggregates, as previously reported to occur in acidic solutions of p-FTAA and other similar COEs. ${ }^{[8,27]}$ At later times (after $~ 90$ minutes of heating), when pre-fibrillar states of insulin are expected to be present in the solution, this shoulder gradually blends into a more smoothly decaying auto-correlation function, a consequence of growing, broader size-distributions. This indicates a complex situation possibly showing that the formation of pre-fibrillar species destabilizes and breaks down the p-FTAA aggregates, followed by association of p-FTAA onto pre-fibrillar states of insulin, yielding the successively diminishing shoulder in the correlation function.

Surprisingly, continued heating does not extend the auto-correlation function to longer diffusion times, but generates curves similar to that for pure insulin after 30 minutes of heating.

It should be noted that the angular dependency of Mie scattering from particles larger than the incident wavelength, makes the CONTIN distribution a less than ideal quantitative measure of sizes represented in the sample as the distribution is displaced toward smaller dimensions. In the CONTIN analyses shown in figure $2 b$, data are weighted against scattering intensity which in turn displaces the distribution toward larger dimensions. To perform a quantitative CONTIN size analysis of the two samples multiple measurements from different angles are necessary, which is not possible with the available instrument setup. However, if we disregard these complications and just compare the distributions and correlation functions from the two fibrillation events qualitatively, it is clear that the kinetics of the early stage fibrillation event is affected by the presence of p-FTAA in the sample.

We used TEM to confirm and further elucidate the DLS results. Comparing TEM micrographs of insulin dispersions after 150 minutes heating reveals severe geometrical differences, possibly caused by the presence of p-FTAA. The images for BI alone show fibrillike nanostructures (Figure 3a, b); $\sim 10 \mathrm{~nm}$ in width and several $\mu \mathrm{m}$ in length similar to those previously reported in literature. ${ }^{[4-6]}$ With p-FTAA present during heating, the number of fibril-like structures is reduced (Figure 3c, d). Instead, a short coarse structure is observed, with a length of 50 to $100 \mathrm{~nm}$ and a thickness around $15 \mathrm{~nm}$. Apparently, the process of fiber growth is perturbed by the presence of p-FTAA, possibly favoring collateral growth behavior during the earlier stage of amyloid formation. This more coarse structure fits quite well with the difference in representation of size distribution in the DLS auto-correlation function discussed above (Figure 2a).

Next, we followed the early phase of the fibrillation process of insulin with fluorescence spectroscopy. Distinct differences were observed in the fluorescence from samples where pFTAA was mixed with samples removed from the fibrillation process, compared to if the p- 

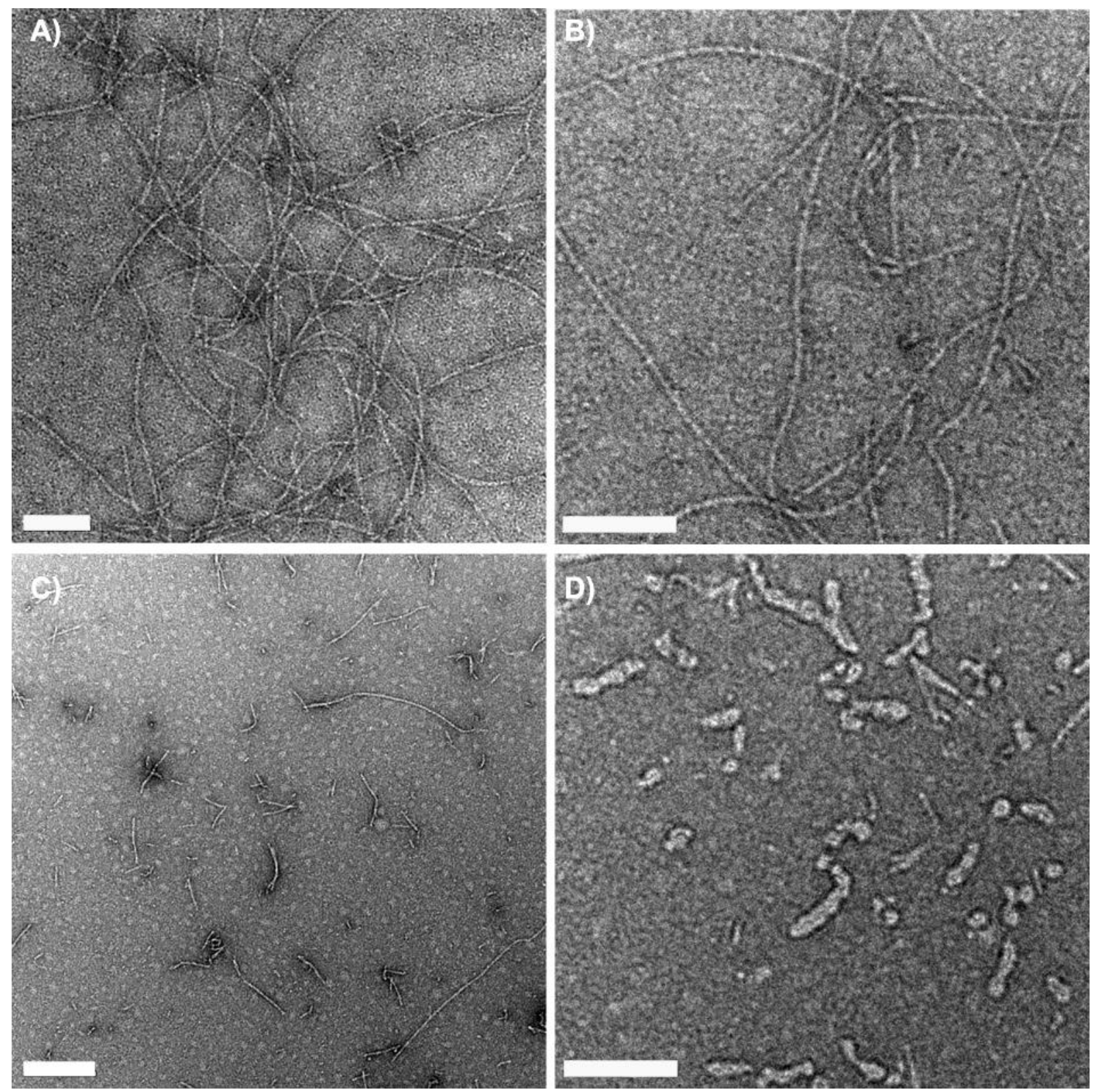

Figure 3. TEM images of insulin fibers captured after 150 minutes of heating, without (A, B) or in the presence of p-FTAA (C, D) during the fibrillation process, (Scale bars; $200 \mathrm{~nm}$ in panel $(\mathrm{C}), 100 \mathrm{~nm}$ in the others)

FTAA was present during heating of the insulin sample (Figure 4a). This is in contrast to what has previously been reported for fibrillation of both $A \beta$ peptide ${ }^{[9]}$ as well as $B{ }^{[18]}{ }^{[n}$ these studies p-FTAA changed its fluorescence spectral signature after only a few minutes of heating reaching a lag phase and continued to change after 20 minutes of heating. This second shift coincides with the point where thioflavin-T (Th-T) fluorescence intensity starts to increase when following the fibrillation kinetics. It may be tempting to explain our result as pre-aggregation of BI in the sample also containing p-FTAA. However, the excitation spectra (from the same samples) following the two emission peaks (515 and $545 \mathrm{~nm}$ ), which changed almost identically during the fibrillation process regardless of whether p-FTAA was present or absent during heating (Figure $4 \mathrm{~b}$ ), contradict this interpretation. Moreover, the excitation spectra from p-FTAA displayed distinct differences between the initial unfolded/native state before the fibrillation process and the pre-fibrillar states that appeared during heating (Figure 4b). A large red-shift was observed already after 15 minutes of heating, and continued to red- 

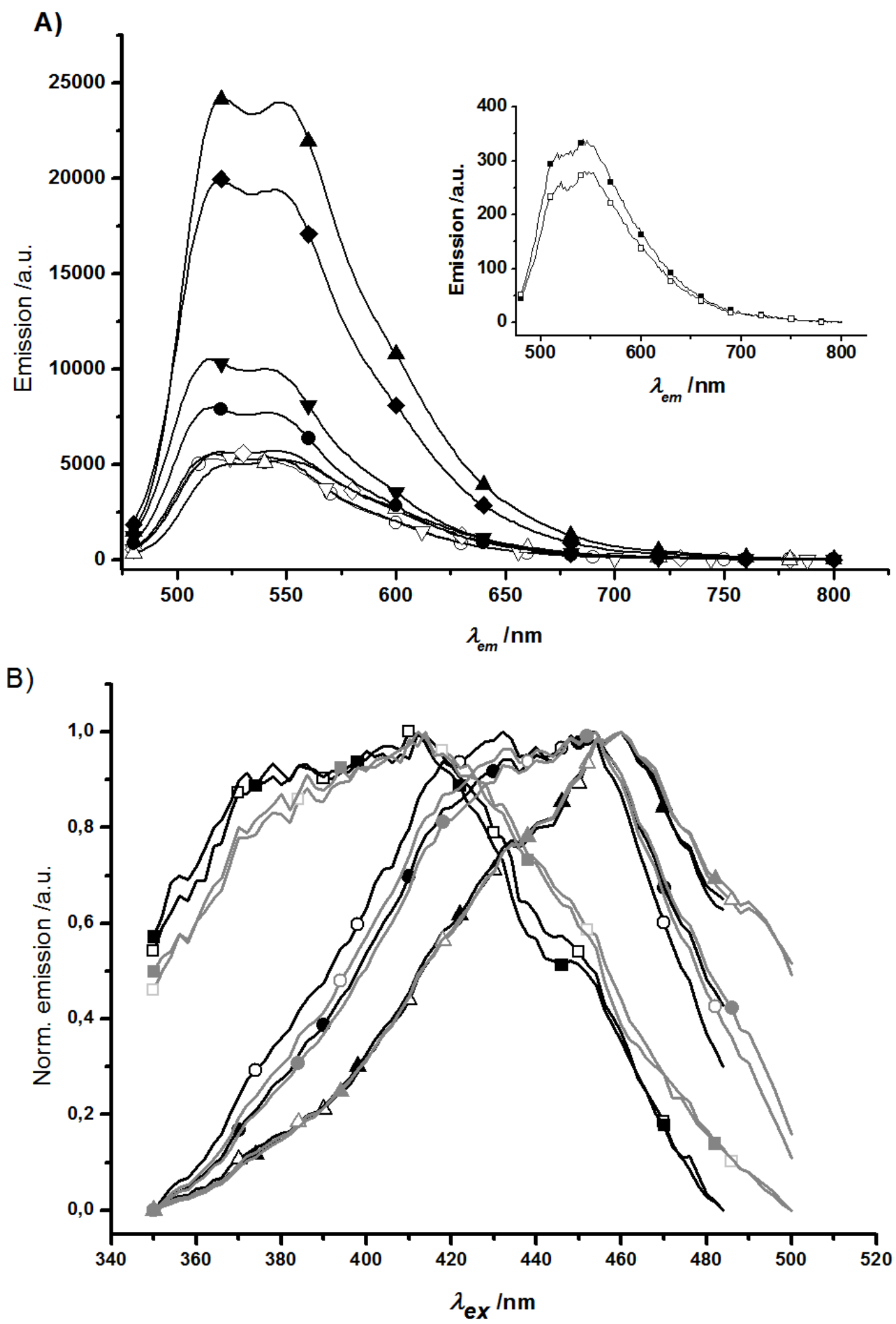

Figure 4. Emission spectra of p-FTAA when excited with $450 \mathrm{~nm}$ (A) and corresponding excitation spectra (B) with emission measured during the fibrillation process at $515 \mathrm{~nm}$ (black) and $545 \mathrm{~nm}$ (grey) respectively. Inset figure 4a: Corresponding emission spectra before heating started. Solid symbols denote measurements with p-FTAA present during heating, open symbols without p-FTAA present during the heating: $(\bullet, \circ) 15$ minutes, $(\nabla, \nabla)$ 45 minutes, $(\diamond, \diamond) 90$ minutes and $(\boldsymbol{\Delta}, \triangle) 120$ minutes after the heating process started. 
shift during the fibril formation process. The peak became more distinct, indicating different excitation pathways for p-FTAA in insulin solution, and when bound to different fibrillar states of insulin. We suggest that this large red shift derives from a conformation change of the COE backbone, extending the effective conjugation length and hence the magnitude of the transition dipole moment along the COE backbone in this spectral range. The two emission peaks $(515 \mathrm{~nm}$ and $545 \mathrm{~nm}$ ) originate from the same relaxation process, presumably the vibronic manifold of an ordered p-FTAA structure.

The growth of insulin amyloid fibers in the absence or presence of p-FTAA was further investigated by fluorescence correlation spectroscopy (FCS). The samples showed distinctly different behavior already after 10 minutes of heating. The pure insulin sample, with p-FTAA added after heating, exhibited a smooth fluorescence intensity trace (Figure 5a), with a mean intensity of $\sim 2.1 \mathrm{kHz}$. In contrast, when p-FTAA had been present during heating, the average fluorescence intensity was higher $(\sim 10.3 \mathrm{kHz})$ and a number of spikes were visible in the trace. This tendency became even more pronounced after 40 minutes of heating. The mean intensity increased to $\sim 3.4 \mathrm{kHz}$ in the insulin sample and to $\sim 20.5 \mathrm{kHz}$ in the insulin p-FTAA sample, the latter with increased fluctuations. We attribute the fluorescence intensity spikes in the traces to growing, intensely emitting diffusing units. The very different traces clearly show that, if p-FTAA is present during the fibrillation process, complexes with a wide spread of fluorescence intensities are formed in the pre-fibrillar state. The maximum brightness of these complexes is much higher than of those formed if p-FTAA is added after the insulin dispersion has been cooled down. Without p-FTAA present, the growth phase shows a much more homogenous development, generating fluorescence traces containing fewer spikes. The quadratic intensity dependence of the auto-correlation function ${ }^{[28]}$ makes a detailed analysis of the corresponding auto-correlation curves (Figure 5b) very error-prone. It is however clear that the complexes contributing the most to the correlation curves, i.e. gives the strongest fluorescence signal, are larger in the samples where p-FTAA was present from the beginning. Continued heating of the samples additionally increased the number and intensity of spikes, making further interpretation of data uncertain.

The data presented above is to some extent inconsistent with what was recently reported in ref $^{[18]}$ where no difference in turbidity/optical density (OD) could be observed with or without p-FTAA present during the fibrillation event. However, dynamic light scattering is a more sensitive method compared to OD, which is reflected by the change in DLS signal already after 15 minutes compared to 60-75 minutes reported with OD. This is approximately 40-55 minutes after the reported detection of fibrillar species with Th-T fluorescence. The higher sensitivity allows observation of early phenomena and smaller objects. They further report a concentration dependent emission spectrum where increased p-FTAA concentration results in less well resolved emission peaks, and a difference in the initial emission which is strongly blue shifted when lowering the concentration from $5 \mu \mathrm{M}$ to $0.05 \mu \mathrm{M}$ of p-FTAA. This indicates larger p-FTAA aggregates for the $5 \mu \mathrm{M}$ solution and possibly single chains when reaching nano-molar levels. p-FTAA has not previously been reported to be found in a single chain conformation. In previous studies it has been concluded that an increased fluorescence emission is correlated to a separation between the emitting COE units as a result of less efficient inter-chain relaxation pathways. ${ }^{[29-31]}$ Our DLS and fluorescence measurement data however, provide clear evidence of large, weakly emitting, diffusing aggregates initially occurring in the insulin/ p-FTAA dispersion, with increasing fluorescence intensity as the supramolecular aggregates continue to grow. Experiments where insulin is left to fibrillate alone, and then mixed with p-FTAA, result in a less pronounced fluorescence change. A likely mechanism is that interactions of p-FTAA with the native insulin create pre-amyloid aggregates incorporating p-FTAA. Continuing growth leads to a spatial separation of adjacent 
p-FTAA molecules, observed as an increased emission in combination with a red-shift of the excitation spectrum (Figure 4). Moreover, according to the FCS data, a more prominent part of the p-FTAA emission originates from larger units when p-FTAA is present during the fibrillation event, as compared to when p-FTAA is added later. This also suggests and fortifies the conclusion that p-FTAA influences the assembly of the pre-fibrillar insulin species.

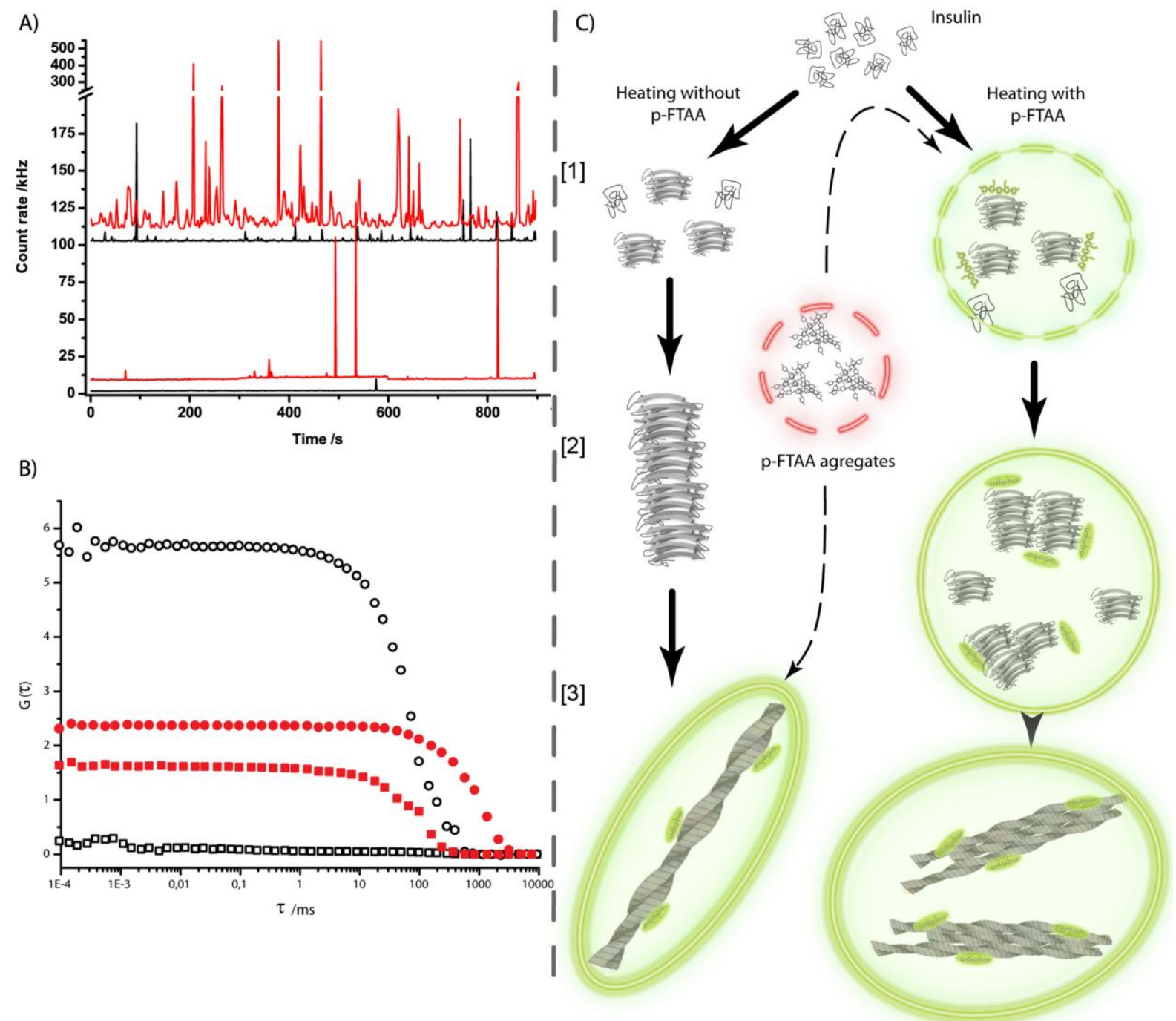

Figure 5. FCS fluorescence intensity trace plots (a) and corresponding auto-correlation function (b) from measurements of insulin with p-FTAA added after the sample was removed from heating (black), and insulin samples with p-FTAA present during heating (red). The stacked intensity traces separated by $100 \mathrm{kHz}$, starting from the bottom, 10 minutes and 40 minutes respectively after heating of the sample to $50^{\circ} \mathrm{C}$ begun. The symbols in the FCS auto-correlation curves represent measurements performed 10 minutes $(\boldsymbol{\square}, \square)$ and 40 minutes $(\bullet, \circ)$, respectively after heating of the sample to $50^{\circ} \mathrm{C}$ begun. (C) Schematic illustration showing the two suggested amyloid formation routes with p-FTAAs present (right) or when introduced after 120 minutes of heating (left). [1] Aggregate formation, [2] prefibrillar structures, [3] thin long pre-amyloid structures (left) or coarser pre-amyloid structures (right). The fluorescence signature and the suggested interactions at the different states are also indicated.

It has been suggested that COEs and other amyloid interacting molecules intercalate in a thin hydrophobic groove along the amyloid fibrils. ${ }^{[15,16,32]}$ Intercalation of p-FTAA in these grooves along the fibril axis might favor a collateral assembly of pre-fibrillar insulin species, 
which would agree with the DLS and FCS measurements, as well as the TEM micrographs showing a fast formation of coarse aggregates in the early fibrillation event. This collateral assembly does not occur when p-FTAA is added afterwards, presumably because many of the binding sites along the pre-fibrillar species are hidden. Visual inspection of our samples at later times, which were not analyzed by FCS or DLS because of precipitation, indicated that the amyloid formation to some extent had occurred even in the samples in which p-FTAA was present, as seen from the formation of a gel. A similar phenomenon, but in the later phase of fibrillation, when mature amyloids have been formed, has also been observed for the zwitterionic COE PONT (also known as tPOWT), ${ }^{[7]}$ where it was shown that the presence of the zwitter-ionic COE during insulin fibrillation lead to formation of higher ordered aggregates of insulin. Also in the presence of PTAA, a higher degree of fibrillar bundling has been reported. ${ }^{[6]}$ This supports our results that the presence of a COE can result in different amyloid assemblies by influencing the amyloid formation.

\section{Conclusion}

From the results presented in this study, it can be concluded that p-FTAA interacts with the pre-fibrillar species of insulin that precede the formation of mature insulin amyloid fibrils. The presence of p-FTAA influences the fibrillation growth process, and favors a collateral assembly of the pre-fibrillar species. Apparently, the molecular interplay of pre-fibrillar insulin species and p-FTAA is complex, with the different species influencing each other. This study supports the tantalizing possibility of using p-FTAA as a possible diagnostic or therapeutic means for detecting or even influencing formation of pre-amyloid assemblies, or as a functional luminescent building block for nanofabrication. We further show that the two emission peaks of p-FTAA associated with amyloid formation reported in the literature is closely related. They exhibit very similar behavior, regardless of whether p-FTAA is present during the fibrillation process or added to a sample after heating, indicating that they are results of similar excitation and relaxation pathways.

Acknowledgements: The author's wish to thank Dr. Daniel Aili, Linköping University for assistance with the TEM micrographs, Dr. K. Peter R. Nilsson, Linköping University for kindly supplying the p-FTAA and for valuable comments and discussion. We thank Dr. Thomas Ederth, Linköping University for valuable consultation and interpretation of DLS data and Dr. Fredrik Westerlund, Chalmers technical university for valuable comments. This study was supported by the Swedish Science Council (VR), and the Strategic Research Foundation (SSF) through the center for organic bioelectronics (OBOE). We thank the Knut and Alice Wallenberg foundation for instrument grants.

Received: ((will be filled in by the editorial staff)); Revised: ((will be filled in by the editorial staff)); Published online: DOI: 10.1002/mabi.201100016

Keywords: biofibers, conjugated polymers, fluorescence, light scattering, nano composites.

[1] P. Björk, A. Herland, M. Hamedi, O. Inganäs, J. Mater. Chem. 2010, 20, 2269.

[2] C. M. Dobson, Nature 2003, 426, 884.

[3] L. C. Serpell, M. Sunde, C. C. F. Blake, Cell. Mol. Life Sci. 1997, 53, 871. 
[4] J. D. Harper, C. M. Lieber, P. T. Lansbury, Chem. Biol. 1997, 4, 951.

[5] C. L. Shen, G. L. Scott, F. Merchant, R. M. Murphy, Biophys. J. 1993, 65, 2383.

[6] A. Herland, P. Björk, P. R. Hania, I. G. Scheblykin, O. Inganäs, Small 2007, 3, 318.

[7] A. Herland, K. P. R. Nilsson, J. D. M. Olsson, P. Hammarström, P. Konradsson, O. Inganäs, J. Am. Chem. Soc. 2005, 127, 2317.

[8] A. Åslund, A. Herland, P. Hammarström, B.-H. Jonsson, K. P. R. Nilsson, O. Inganäs, P. Konradsson, Bioconjugate Chem. 2007, 18, 1860.

[9] A. Åslund, C. J. Sigurdson, T. s. Klingstedt, S. Grathwohl, T. Bolmont, D. L. Dickstein, E. Glimsdal, S. Prokop, M. Lindgren, P. Konradsson, D. M. Holtzman, P. R. Hof, F. L. Heppner, S. Gandy, M. Jucker, A. Aguzzi, P. HammarstroÌ’m, K. P. R. Nilsson, ACS Chem. Biol. 2009, 4, 673.

[10] M. Hamedi, A. Herland, R. H. Karlsson, O. Inganäs, Nano Lett. 2008, 8, 1736.

[11] J. Wigenius, P. Bjork, M. Hamedi, D. Aili, Macromol. Biosci. 2010, 10, 836.

[12] M. Hamedi, J. Wigenius, F. I. Tai, P. Bjork, D. Aili, Nanoscale 2010, 2, 2058.

[13] H. Tanaka, A. Herland, L. J. Lindgren, T. Tsutsui, M. R. Andersson, O. Inganäs, Nano Lett. 2008, 8, 2858.

[14] S. Barrau, F. Zhang, A. Herland, W. Mammo, M. R. Andersson, O. Inganäs, Appl. Phys. Lett. 2008, 93.

[15] J. Wigenius, M. R. Andersson, E. K. Esbjörner, F. Westerlund, Biochem. Biophys. Res. Commun. 2011, 408, 115.

[16] A. Herland, O. Inganäs, Macromol. Rapid Commun. 2007, 28, 1703.

[17] B. Vestergaard, M. Groenning, M. Roessle, J. S. Kastrup, M. van de Weert, J. M. Flink, S. Frokjaer, M. Gajhede, D. I. Svergun, PLoS Biol. 2007, 5, 1089.

[18] P. Hammarström, R. Simon, S. Nyström, P. Konradsson, A. Åslund, K. P. R. Nilsson, Biochemistry 2010, 49, 6838.

[19] J. Hardy, D. J. Selkoe, Science 2002, 297, 353. 
[20] R. Kayed, E. Head, J. L. Thompson, T. M. McIntire, S. C. Milton, C. W. Cotman, C. G. Glabe, Science 2003, 300, 486.

[21] C. G. Glabe, NeuroBiol. Agi. 2006, 27, 570.

[22] S. W. Provencher, Comput. Phys. Commun. 1981, 27, 213.

[23] D. Magde, E. L. Elson, W. W. Webb, Biopolymers 1974, 13, 29.

[24] H. Vogel, L. Nilsson, R. Rigler, S. Meder, G. Boheim, W. Beck, H. H. Kurth, G. Jung, Eur. J. Biochem. 1993, 212, 305.

[25] J. Widengren, U. Mets, R. Rigler, J. Phys. Chem 1995, 99, 13368.

[26] R. Hanbury- Brown, R. Q. Twiss, Nature 1956, 177, 27.

[27] J. Wigenius, Doctoral Thesis, Linköping University (Linköping, Sweden), May, 2010.

[28] E. L. Elson, D. Magde, Biopolymers 1974, 13, 1.

[29] F. C. Spano, J. Chem. Phys. 2005, 122.

[30] J. Clark, C. Silva, R. H. Friend, F. C. Spano, Phys. Rev. Lett. 2007, 98.

[31] P. Björk, D. Thomsson, O. Mirzov, J. Wigenius, O. Inganäs, I. G. Scheblykin, Small 2009, 5, 96 .

[32] M. Groenning, M. Norrman, J. M. Flink, M. van de Weert, J. T. Bukrinsky, G. Schluckebier, S. Frokjaer, J. Struct. Biol. 2007, 159, 483.

[33] A. Porollo, J. Meller, BMC Bioinformatics 2007, 8, 316.

A conjugated oligoelectrolyte (COE) can detect misfolded insulin, and also influence the kinetics of the early stages of amyloid formation. The growth of structures formed during misfolding of insulin has been followed by light scattering, fluorescence correlation spectroscopy and electron microscopy. A marked influence of the COE on the kinetics of amyloid growth may have relevance for theranostics.

Jens Wigenius, Gustav Persson, Jerker Widengren and Olle Inganäs* 
Interactions between a luminescent conjugated oligoelectrolyte and insulin during early phases of amyloid formation 